\title{
Analysis of Maceaene and Macamide Contents of Petroleum Ether Extract of Black, Yellow, and Purple Lepidium Meyenii (Maca) and Their Antioxidant Effect on Diabetes Mellitus Rat Model
}

\author{
Congyang Qiu ${ }^{1,2}$, Tonglin Zhu ${ }^{1 *}$; Liying Lan ${ }^{2}$, Qingmao Zeng ${ }^{1}$ and Zhiguo Du ${ }^{1}$. \\ ${ }^{1}$ College of Information, South China Agricultural University, Guangzhou, China. ${ }^{2}$ College of Applied Science, Jianxi \\ University of Science and Technology, Ganzhou, Jiangxi, China.
}

\begin{abstract}
Maceaene and macamide contents as well as antioxidant effect of petroleum ether extract of black maca (BM), yellow maca (YM), and purple maca (PM) on diabetes mellitus (DM) rats were investigated. The results showed that seven, six, and five analogues of macamides were identified from the petroleum ether extracts of BM, YM, and PM, respectively. BM extract exhibited the highest contents of total macamides. Comparatively, the PM extract has the lowest macamide quantity. The maceaene contents in all the extracts showed no significant difference ( $p>0.05)$. Macamide contents in maca with the same color were not statistically different. Pharmacological results showed that 60-day oral administration of the petroleum ether extract of maca $(100 \mathrm{mg} / \mathrm{kg} . \mathrm{d})$ can significantly decrease lipid oxidation as indicated by the decreased thiobarbituric acid reactive substances (TBARS) and carbonylated proteins (CP) concentrations on DM rat model $(\mathrm{P}<0.05)$. Among them, oral administration of PM extract showed the lowest TBRAS and $\mathrm{CP}$ concentrations. All maca extracts can enhance antioxidant enzyme (SOD, superoxide dismutase; CAT, catalase) activity of liver and red blood cells (RBC) of DM rat. However, only oral administration of PM extract can increase SOD and CAT activity of both RBC and liver. The glutathion (GSH) contents in plasma were significantly increased in DM rats treated with PM extract $(\mathrm{p}<0.05)$. But, oral administration of BM and YM extracts did not enhance GSH levels. Take together, the data suggested that PM extract exhibited the most potent antioxidant activity on DM rat model. And, maceaene and macamide in maca extract was not correlated with its antioxidant ability.
\end{abstract}

Key words: maca extract, antioxidant activity, maceaene, macamide, diabetes mellitus rat

\footnotetext{
1'Authors for correspondence: 569256786@qq.com
} 


\section{INTRODUCTION}

In diabetes mellitus (DM), chronic hyperglycaemia produces multiple biochemical sequelae, and diabetes-induced oxidative stress could play a role in the symptoms and progression of the disease [1]. Oxidative stress may result in overproduction of oxygen free-radical precursors and/or decreased efficiency of the antioxidant system [2]. The oxygen free-radical generation is associated with auto-oxidation of glucose, impaired glutathione metabolism, alterations in the antioxidant enzymes and formation of lipid peroxides [3-5]. There are various endogenous defense mechanisms against free radicals, such as the enzymes GSH, SOD, GPx, and CAT, whose activities eliminate superoxide, hydrogen peroxide and hydroxyl radicals [6]. Oxidative stress is increased in experimental models of streptozotocin (STZ)-induced diabetes mellitus [7].

Maca (Lepidium meyenii Walp. synonym, $L$. peruvianum Chacon, Brassicaceae), a root crop similar to radish (Raphanus sativus L.), has been consumed in Peru for thousands of years as food and medicine [8]. The plant is originally used to increase human and livestock stamina and to ameliorate fertility problems associated with living at the high elevations where the plant grows [9]. The reputation of maca as a fertility enhancer has increased the popularity of the plant in the USA and other Western countries [10]. Recent studies [1115] have confirmed the effectiveness of maca as a libido and fertility enhancer. Maca, however, has many other traditional therapeutic uses, e.g. as a laxative and for the treatment of rheumatism, respiratory problems, premenstrual discomfort, and menopausal symptoms [10]. Consumption of maca is also reputed to regulate hormone secretion, stimulate metabolism, improve memory and combat depression, anaemia, leukaemia, AIDS, cancer and alcoholism [16]. Even with the reputation of maca as a panacea, scientific research has primarily been focused on its properties of sexual stimulus. Maca contains several kinds of secondary metabolites $[17,18]$, of which macaene and macamides were mainly and uniquely two types of secondary metabolites in this plant and were often thought to be related its pharmacological properties [11]. It has been observed that maca batches from different producers significantly vary in the amount of macaene and macamides [19-21]. More recently, it was revealed that maca with different skin colors was pharmacologically different [22], which may be led due to the diversity of secondary metabolites .

Yunnan is a part of Yun-Gui plateau with an average altitude of more than $2000 \mathrm{~m}$. Maca, as a herbal medicine, was widely cultivated in the region. The skin colors of maca in the region were mainly black, yellow, and purple. The purpose of this study is to analyze the macaene and macamides of petroleum ether extract of maca in Yunnan Province and evaluate their antioxidant effect on DM rat model.

\section{MATERIALS AND METHODS}

\section{Materials}

Acetonitrile, reagent methanol, petroleum ether, and trifluoroacetic acid were HPLC grade and purchased from Fisher (USA). Authentic compounds were purchased from Institute of Processing Engineering, Chinese Academy of Sciences (Beijing, China). Identity and purity of each compound were confirmed by chromatographic (TLC and HPLC) and spectroscopic methods (IR, 1D- and 2D-NMR, high resolution electrospray ionization mass spectroscopy (HR-ESI-MS)). Streptozotocin, citrate, and 2-(4-iodophenyl)-3-(4-nitrophenol)-5phenyltetrazolium chloride were purchased from sigma Co.. Nine samples of maca (three samples each color) were collected in Traditional Chinese Medicine Market of Juhua Village in Kunming City, Yunnan Province, on 8, Sep., 2014. Three samples with black skin were designated as BM1, BM2 and BM3, In a similar way, YM1, YM2, YM3 and PM1, PM2, PM3 referred to maca with yellow and purple skins, respectively.

\section{Sample Preparation}

A $1.0 \mathrm{~kg}$ portion of the dried plant material was extracted three times with $4.5 \mathrm{~L}$ of methanol by sonication for $5 \mathrm{~h}$. The extract was evaporated to get a residue (78-92 g) under reduced pressure, which was suspended in water $(80 \mathrm{ml})$ and then partitioned with petroleum ether. Petroleum ether extract was evaporated to get a dried residue (28-47g). Petroleum ether extract $(1 \mathrm{~g})$ were dissolved in 50 $\mathrm{ml}$ methanol/ $\mathrm{H}_{2} \mathrm{O}(8: 2, \mathrm{v} / \mathrm{v})$ for HPLC-MS analysis. The remaining extract was used for animal experiment. 


\section{Analytical method}

HPLC experiments were performed on a Waters Alliance 2690 HPLC system equipped with a 996 photodiode array detector (Waters, Milford, MA, U.S.A.). A Synergi MAX-RP 80Å column (15034.6 $\mathrm{mm}, 4 \mathrm{~mm}$ particle size) from Phenomenex (Torrance, CA, U.S.A.) was used for all separations. The mobile phase consisted of $\mathrm{H}_{2} \mathrm{O}(\mathrm{A})$ and acetonitrile (B), both containing $0.025 \%$ trifluoroacetic acid (TFA). Separations were performed by linear gradient elution from $45 \mathrm{~A} / 55 \mathrm{~B}$ to $5 \mathrm{~A} / 95 \mathrm{~B}$ during $35 \mathrm{~min}$. The flow rate was adjusted to $1.0 \mathrm{ml} / \mathrm{min}$, with detection wavelengths of $210 \mathrm{~nm}$ and $280 \mathrm{~nm}$, respectively. The column oven was set to $40^{\circ} \mathrm{C} .10 \mathrm{ml}$ of sample was injected for singlet analysis by automatic sampler. Peaks were assigned by spiking the samples with standard compounds, and comparison of the UV-spectra and retention time. All data was recorded and processed by Millennium 32 software from Waters.

\section{Induction of experimental diabetes}

Ninety-day-old Wistar rats, were given a norm caloric standard diet (Hindustan Lever, Kolkata, India) and deionized water ad libitum, while being maintained in a controlled environment (12 h light and dark cycle, $21-23^{\circ} \mathrm{C}$ ). The animals were acclimatized to the laboratory conditions for one week before the start of the experiment. All experiments were conducted in accordance with the NIH14 prescriptions. STZ was freshly dissolved in citrate buffer $(0.01 \mathrm{M}, \mathrm{pH} 4.5)$ and maintained on ice. Diabetes was induced with a single i.p. injection of STZ $(50 \mathrm{mg} / \mathrm{kg})$ to overnight fasted rats. Control rats were injected with citrate buffer. Diabetic status was confirmed in the STZ-treated rats by measuring the fasting plasma glucose after $72 \mathrm{~h}$. Blood $(0.5 \mathrm{ml})$ was collected into heparanised tubes by puncturing the retro-orbital plexus. The plasma was centrifuged at $2000 \mathrm{G}$, for $10 \mathrm{~min}$. After removing the buffer coat, the packed red blood cells (RBC) were washed twice with cold isotonic physiological saline solution. Then a known volume of RBCs was lysed in cold phosphate buffer $(\mathrm{pH}=7.4)$. The haemolysate was separated by centrifuging at $3000 \mathrm{G}$, for $10 \mathrm{~min}$, at $2^{\circ} \mathrm{C}$. Both plasma and haemolysates were used for biochemical analysis. Glucose level was estimated using a commercial glucose kit (Qualigens Diagnostics-Accu-Chek- ROCHE). Rat with plasma glucose levels above $14.82 \mathrm{mmol} / \mathrm{L}$ were considered as diabetic and used in the subsequent experiments.

\section{Experimental design}

One hundred (diabetic) and ten (normal) rats divided into eleven groups of 10 animals each were used to investigate the antioxidant effect of maca extracts: normal control group (N-CK), diabetic control group (D-CK), BM1 group (diabetic +100 $\mathrm{mg} / \mathrm{kg} . \mathrm{d}$ BM1), BM2 group (diabetic +100 $\mathrm{mg} / \mathrm{kg} . \mathrm{d}$ BM2), BM3 group (diabetic +100 mg/kg.d BM3), YM1 group (diabetic +100 $\mathrm{mg} / \mathrm{kg} . \mathrm{d}$ YM1), YM2 group (diabetic +100 $\mathrm{mg} / \mathrm{kg}$.d YM2), YM3 group (diabetic +100 $\mathrm{mg} / \mathrm{kg} . \mathrm{d}$ YM3), PM1 group (diabetic $+100 \mathrm{mg} / \mathrm{kg} . \mathrm{d}$ PM1), PM2 group (diabetic $+100 \mathrm{mg} / \mathrm{kg} . \mathrm{d} \mathrm{PM} 2$ ), and PM3 group (diabetic $+100 \mathrm{mg} / \mathrm{kg} . \mathrm{d} \mathrm{PM} 3$ ). The extracts were suspended in CMC $(0.05 \mathrm{~g} / \mathrm{ml})$ and orally administered via an intragastric tube (2 $\mathrm{ml} / \mathrm{rat}$ ) on a daily basis for 60 days. N-CK and D$\mathrm{CK}$ rats were received $\mathrm{CMC}$ alone. After the last treatment, all the rats were fasted overnight and sacrificed by cervical decapitation. For each group, levels of plasmatic glucose were determined at the beginning of the experiment, 96 hours after STZ injection and at the end of the experiment. The study was approved by the Ethics Committee of South China Agricultural University(Guangzhou, China) and conformed to the standards set by the Guangdong Experimental Animal Management Board.

\section{Tissue collection and processing}

Animals were sacrificed by decapitation after blood collection. Both testes were immediately removed and placed in a Petri dish. After a brief wash in cold saline, fats connective tissues and blood vessels were removed. The liver was homogenized by using a Potter-Elvejham homogenizer with physiological serum, and centrifuged at 5,000 rpm, then the supernatants were collected for the measurement of biochemical parameters. .

\section{Thiobarbituric acid reactive substances assay} Frozen testes were homogenized in $0.1 \mathrm{M}$ TrisHCL buffer $(\mathrm{pH}=7.5)$ at a ratio of $1: 4$ (weight:volume), using a Potter-Elvehjem homogenizer at $4^{\circ} \mathrm{C}$. The formation of thiobarbituric acid reactive substances (TBARS), as an indicator of lipid peroxidation, in the homogenate was assessed by the method described by Ohkawa et al [23]. In brief, a $3 \mathrm{~mL}$ reaction mixture containing $0.1 \mathrm{~mL}$ of liver tissue homogenate, $0.2 \mathrm{~mL}$ of sodium dodecyl sulfate, 1.0 $\mathrm{mL}$ of acetic acid $(\mathrm{pH}=3.5$, adjusted with $1 \mathrm{M}$ $\mathrm{NaOH}), 1.5 \mathrm{~mL}$ of aqueous solution of thibarbituric 
acid (TBA), and $0.7 \mathrm{~mL}$ water was prepared. After heating at $95^{\circ} \mathrm{C}$ for one hour in a hot water bath and cooling, $1 \mathrm{~mL}$ of distilled water and $5 \mathrm{~mL}$ mixture of $\mathrm{n}$-butanol and pyridine (15:1, v/v) were added. Then, the mixture was shaken vigorously on a vortex mixer and was centrifuged at 3,000 rpm for 10 minutes. The absorbance of the upper organic layer was read at $532 \mathrm{~nm}$.

\section{Endogenous antioxidant activity assessment}

Superoxide dismutase (SOD) activities of RBC and liver tissues were measured using commercials kits from Randox Laboratories Ltd (London, UK). One unit of SOD was defined as the amount that caused a $50 \%$ inhibition rate of reduction of 2-(4iodophenyl)-3-(4-nitrophenol)-5-

phenyltetrazolium chloride (INT). Activity of SOD was expressed in $\mathrm{U} / \mathrm{mg}$. $\mathrm{Hb}$ or $\mathrm{U} / \mathrm{mg}$. prot. Catalase activity (CAT) was determined using a commercial kit K773-100 from Biovision Inc. (Milpitas, CA, USA). The activity of CAT was expressed as in $\mathrm{U} / \mathrm{mg}$. $\mathrm{Hb}$.

\section{Reduced glutathione (GSH) assay}

GSH levels in plasma were measured using commercials kits from Randox Laboratories Ltd (London, UK).The GSH contents were expressed in $\mathrm{mg} / \mathrm{g}$.

\section{Carbonylated proteins (CP) assay}

$1 \mathrm{ml}$ liver tissue homogenate is diluted with $4 \mathrm{ml}$ phosphate-buffered saline (PBS), containing 10 $\mathrm{mM}$ sodium phosphate and $0.15 \mathrm{M} \mathrm{NaCl}(\mathrm{pH}=$ 7.4). And this was centrifuged twice $(10 \mathrm{~min}$, $14,000 \mathrm{rpm}$ ) in a tabletop microcentrifuge to eliminate all particulate matter. The diluted proteins are precipitated with $0.5 \mathrm{ml}$ cold trichloroacetic acid (TCA, 20\% final concentration) and then collected by centrifugation $(25000 \mathrm{rpm})$ for $5 \mathrm{~min}$. $0.5 \mathrm{ml} \mathrm{DNPH}$ (dinitrophenylhydrazone $10 \mathrm{mM}$ ) in $2 \mathrm{~N} \mathrm{HCl}$ is added to the protein pellet to give a final protein concentration of $1-1.5 \mathrm{mg} / \mathrm{ml}$. Samples are allowed to stand in the dark room for $1 \mathrm{~h}$ with vortexing every $10 \mathrm{~min}$; they are then precipitated with $15 \%$ TCA (final concentration) and centrifuged for $5 \mathrm{~min}$. The supernatants are discarded, and the protein pellets are washed three times with 15\% TCA, and then was washed three times with $1 \mathrm{ml}$ ethanol/ethyl acetate $(1: 1, \mathrm{v} / \mathrm{v})$ to remove free DNPH. Samples are then resuspended in $2 \mathrm{ml} 6 \mathrm{M}$ guanidine hydrochloride $(\mathrm{GdmCl}$, dissolved in $2 \mathrm{~N} \mathrm{HCl}, \mathrm{pH}=2.3$ ) at $37^{\circ} \mathrm{C}$ for $15 \mathrm{~min}$ with vortex mixing. Carbonyl contents are determined from the absorbance at $366 \mathrm{~nm}$. The results were expressed in nmol per mg protein.

\section{Statistical analysis}

Statistical analysis was performed by 2-way ANOVA using GraphPad Prism (GraphPad Software Inc., La Jolla, CA, USA) Version 6. To identify the sources of significant main effect, post hoc comparisons (Tukey's t test) were used. Data were expressed as mean \pm standard deviation (SD), and statistical significance was assigned at $p \leq 0.05$.

\section{RESULTS}

\section{Macamide and maceaene contents in petroleum ether extract of BM, YM, and PM}

Macamide variations and contents in maca from different origin or with different colors differed vastly. More than 10 analogues of macamides have been identified from the maca extract [24-27]. To quantify macamide contents in maca plant, the first and key step is to ascertain the variations of macamides. Therefore, HPLC-MS was used to scan the extract of BM, YM, and PM. The results showed BM contained seven macamides, YM and PM contained six and five analogues of macamides, respectively. $\mathrm{N}$-benzylpalmitamide was only found in BM extract. All the identity of macamides was further confirmed by comparison of retention time with those of standard compounds. The contents of macamides range from 1.47 to $15.03 \mathrm{ng} / \mathrm{g}$.DW. The YM extract showed the highest total contents of macamides (average 45.85, BM1-BM3; average 57.49, YM1-YM3; average 41.50, PM1-PM3). And the macamide contents in BM extract was inbetween. Maceaene in all the nine samples can be detected and their differentiation of contents was negligible among the samples (Table 1). 
Table 1. Maceaene and macamide contents of BM, YM, and PM.

\begin{tabular}{|c|c|c|c|c|c|c|c|c|c|}
\hline \multirow[t]{2}{*}{ compounds } & \multicolumn{5}{|c|}{ Contents (ng/g. DW) } & \multirow[b]{2}{*}{ YM3 } & \multirow[b]{2}{*}{ PM1 } & \multirow[b]{2}{*}{ PM2 } & \multirow[b]{2}{*}{ PM3 } \\
\hline & BM1 & BM2 & BM3 & YM1 & YM2 & & & & \\
\hline N-Benzylhexadecanamide & $12.3 \pm 2.11$ & $12.54 \pm 1.37$ & $14.12 \pm 1.59$ & $\mathrm{ND}$ & ND & $\mathrm{ND}$ & $4.38 \pm 0.58$ & $5.07 \pm 0.65$ & $4.66 \pm 0.44$ \\
\hline $\begin{array}{l}\text { N-Benzyl-5-oxo-6E,8E- } \\
\text { octadecadienamide }\end{array}$ & $1.47 \pm 0.20$ & $1.52 \pm 0.14$ & $1.24 \pm 0.14$ & $3.72 \pm 0.21$ & $4.28 \pm 0.27$ & $3.65 \pm 0.41$ & ND & ND & ND \\
\hline $\begin{array}{l}\mathrm{N}-(3,4-\text { dimethoxybenzyl)- } \\
\text { hexadecanamide }\end{array}$ & ND & ND & ND & $6.94 \pm 0.53$ & $7.31 \pm 1.38$ & $7.02 \pm 0.98$ & $3.68 \pm 0.45$ & $3.50 \pm 0.45$ & $4.12 \pm 0.78$ \\
\hline N-benzil-tetracosanamide & $2.31 \pm 0.10$ & $2.24 \pm 0.34$ & $1.79 \pm 0.23$ & $3.91 \pm 0.42$ & $4.35 \pm 0.43$ & $3.98 \pm 0.23$ & ND & ND & ND \\
\hline oleamide & $3.98 \pm 0.62$ & $4.64 \pm 0.59$ & $3.82 \pm 0.21$ & $12.53 \pm 1.78$ & $13.17 \pm 1.02$ & $12.54 \pm 1.65$ & $6.23 \pm 0.78$ & $5.21 \pm 0.65$ & $6.05 \pm 0.45$ \\
\hline anandamide & $7.92 \pm 0.98$ & $8.07 \pm 1.23$ & $8.57 \pm 1.23$ & $14.87 \pm 2.58$ & $15.03 \pm 1.69$ & $16.11 \pm 1.98$ & $11.53 \pm 1.23$ & $12.95 \pm 1.65$ & $12.39 \pm 0.52$ \\
\hline$N$-benzylpalmitamide & $2.22 \pm 0.17$ & $2.73 \pm 0.11$ & $2.06 \pm 0.31$ & ND & ND & ND & ND & ND & ND \\
\hline$N$-benzyloleamide & $14.70 \pm 1.98$ & $13.92 \pm 2.37$ & $15.40 \pm 1.32$ & $14.20 \pm 0.23$ & $14.59 \pm 1.78$ & $14.28 \pm 2.01$ & $15.63 \pm 1.47$ & $15.17 \pm 1.02$ & $13.93 \pm 1.35$ \\
\hline Maceaene & $20.25 \pm 2.32$ & $19.67 \pm 1.89$ & $21.34 \pm 2.78$ & $22.23 \pm 2.39$ & $20.73 \pm 1.47$ & $20.91 \pm 0.87$ & $21.32 \pm 2.06$ & $21.56 \pm 4.55$ & $20.69 \pm 3.27$ \\
\hline
\end{tabular}

ND: not detected; DW: dried weight.. Average retention time of compounds was as followings: $\mathrm{N}$ benzylhexadecanamide M13.6 min; N-benzyl-5oxo-6E,8E-octadecadienamide $16.8 \mathrm{~min}$; $\mathrm{N}-(3,4-$ dimethoxybenzyl)-hexadecanamide $14.8 \mathrm{~min} ; \mathrm{N}$ benzil-tetracosanamide $18.9 \mathrm{~min}$; oleamide 28.5 min; anandamide $20.7 \mathrm{~min}$; $N$-benzylpalmitamide $29.5 \mathrm{~min} ; \mathrm{N}$-benzyloleamide $32.0 \mathrm{~min}$ and maceaene $34.2 \mathrm{~min}$.

Oral administration of maca extract enhanced the levels of plasma glucose of DM rats Fasting plasma glucose levels were significantly increased in D-CK rats. When treating with maca extracts of $\mathrm{BM}, \mathrm{YM}$, and PM, the levels of plasma glucose of DM rats were significantly decreased and recovered to normal control level. However, maca with different skin colors showed no significantly different effect on the plasma glucose levels (Figure $1)$.

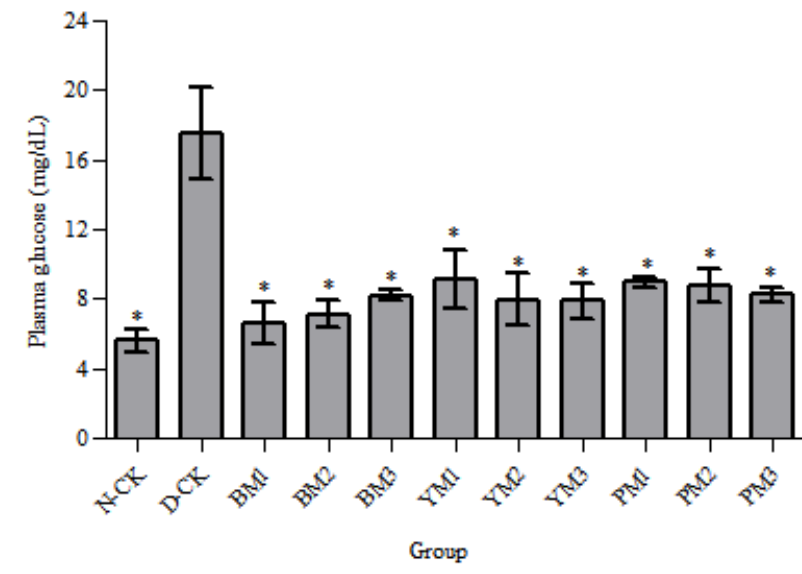

Figure 1. Effect of oral administration of BM, YM, and $\mathrm{PM}$ extracts on plasma glucose of DM rat model
Oral administration of maca extract decreased TBARS and CP contents of liver of DM rat model

TBARS contents of livers were significantly increased after induction of DM in rats. TBRAS levels were significantly decreased after $60 \mathrm{~d}$ oral administration of BM, YM, and PM extracts but still higher than that in N-CK. The TBRAS levels in YM and PM group were decreased to a lower degree when compared with those in BM group. The change of CP of liver was similar to those of TBRAS (Figure 2). All the three maca extracts can decrease CP levels in liver. The decreased levels in YM group were more notable than those in BM and $\mathrm{PM}$ group. The $\mathrm{CP}$ level in $\mathrm{YM}$ group was decreased to an even lower than those in N-CK group (Figure 3).

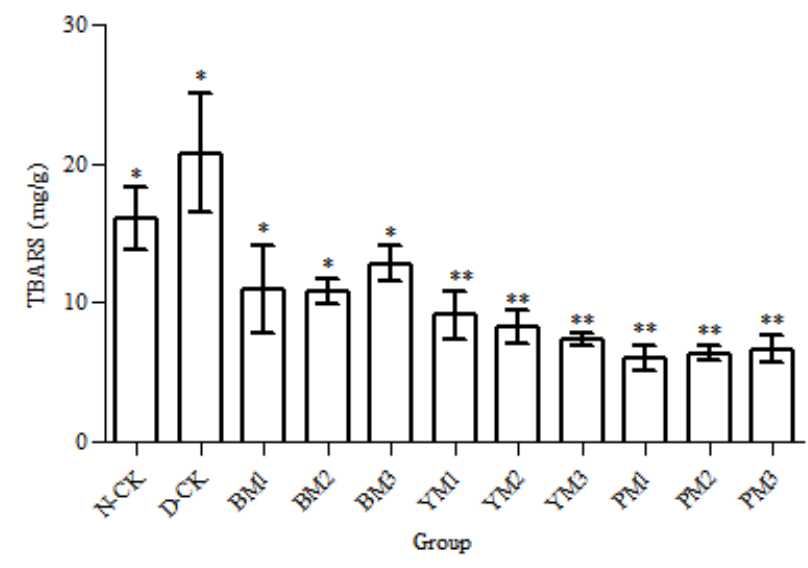

Figure 2. Effect of oral administration of BM, YM, and PM extracts on TBRAS contents of liver of DM rat model 


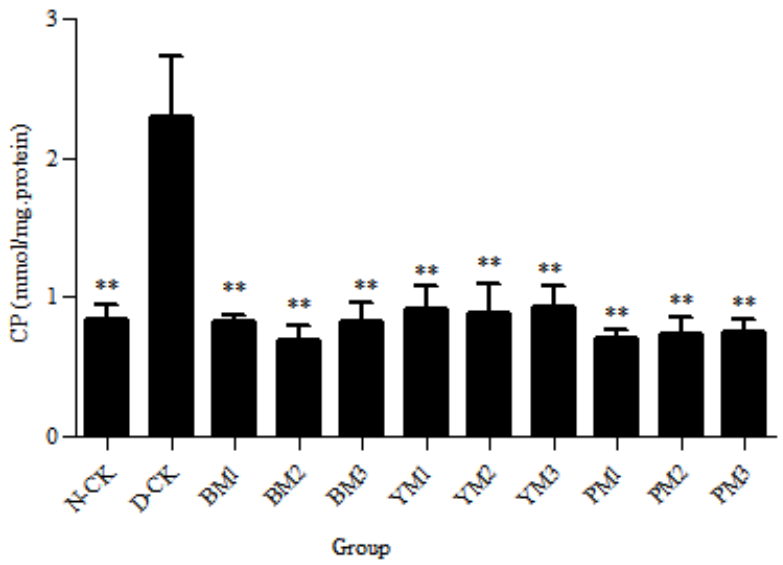

Figure 3. Effect of oral administration of BM, YM, and $\mathrm{PM}$ extracts on the CP contents of liver of DM rat model
Oral administration of PM extract increased SOD and CAT activity of RBC and liver of DM rat model

In the D-CK model, SOD and CAT activity of liver and $\mathrm{RBC}$ were significantly decreased compared with those of N-CK model. The activities of SOD and CAT of RBC and SOD of liver tissues were significantly increased after oral administration of BM extract. YM extract can significantly enhance SOD activity of liver. YM extract did not affect the activities of SOD and CAT of RBC and CAT of liver. While the three PM (PM1-PM3) extracts significantly enhanced SOD and CAT activity of both RBC and liver (Table 2).

Table 2. Effect of oral administration of BM, YM, and PM extracts on SOD and CAT activity of DM rat model

\begin{tabular}{lllll}
\hline Sample & RBC-SOD $(\mathrm{U} / \mathrm{mg} \mathrm{Hb})$ & Liver-SOD $($ Units/mg prot) & RBC-CAT (U/mg Hb) & Liver-SOD (Units/mg prot) \\
\cline { 2 - 5 } N-CK & $7.81 \pm 0.92^{*}$ & $9.23 \pm 1.02^{*}$ & $59.37 \pm 6.52^{*}$ & $5.70 \pm 0.21 \%$ \\
D-CK & $4.23 \pm 0.12$ & $3.55 \pm 0.39$ & $42.20 \pm 5.09$ & $3.03 \pm 0.12$ \\
BM1 & $6.99 \pm 0.45^{*}$ & $3.34 \pm 0.27$ & $63.73 \pm 7.25^{*}$ & $4.07 \pm 0.34^{*}$ \\
BM2 & $6.55 \pm 0.51^{*}$ & $3.27 \pm 0.43$ & $63.21 \pm 8.31^{*}$ & $3.89 \pm 0.20^{*}$ \\
BM3 & $6.76 \pm 0.31^{*}$ & $3.75 \pm 0.52$ & $53.64 \pm 4.17^{*}$ & $4.19 \pm 0.52^{*}$ \\
YM1 & $4.91 \pm 0.72$ & $6.36 \pm 0.21^{*}$ & $52.84 \pm 1.34$ & $3.27 \pm 0.12$ \\
YM2 & $4.38 \pm 0.56$ & $7.17 \pm 0.47^{*}$ & $52.63 \pm 2.03$ & $2.96 \pm 0.14$ \\
YM3 & $4.07 \pm 0.51$ & $7.68 \pm 0.65^{*}$ & $52.57 \pm 4.23$ & $2.94 \pm 0.32$ \\
PM1 & $5.73 \pm 0.17^{*}$ & $5.66 \pm 0.24^{*}$ & $73.86 \pm 9.27^{*}$ & $5.24 \pm 0.41^{*}$ \\
PM2 & $5.45 \pm 0.43^{*}$ & $5.34 \pm 0.23^{*}$ & $69.87 \pm 7.17^{*}$ & $5.42 \pm 0.14^{*}$ \\
PM3 & $5.80 \pm 0.19^{*}$ & $5.23 \pm 0.32^{*}$ & $73.58 \pm 4.08^{*}$ & $5.47 \pm 0.37^{*}$ \\
\hline
\end{tabular}

Oral administration of PM extract enhanced GSH contents in plasma of DM rat model

The GSH contents in plasma of D-CK rat were significantly declined compared with those of $\mathrm{N}$ CK. While, the GSH contents were increased after oral administration of PM extract. Oral administration of $\mathrm{BM}$ and $\mathrm{YM}$ extracts did not affect GSH contents in plasma on DM rats (Figure 4).

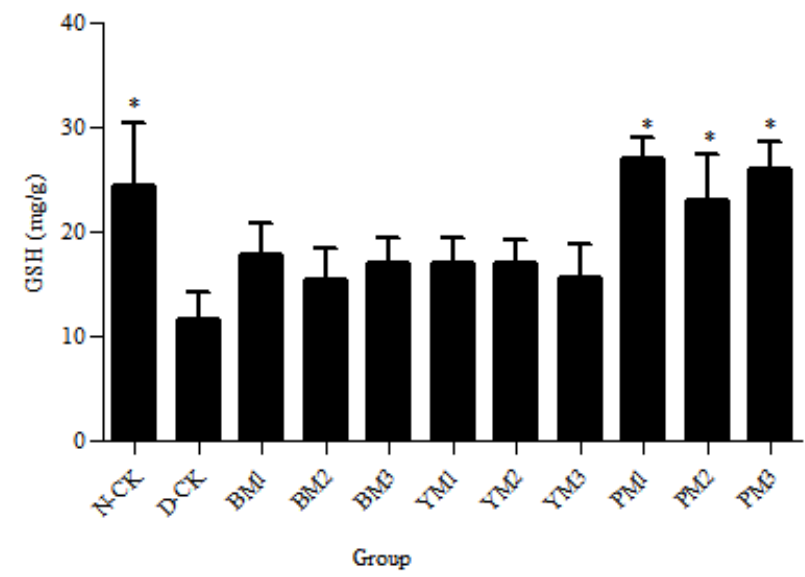

Figure 4. Effect of oral administration of BM, YM, and PM extracts on GSH contents in plasma of DM rat model 


\section{DISCUSSION}

Maca has been thought to be a promising plant recently. However, its pharmaceutical investigation has been focused on increasing fertility ability. Maca quality was different due to its origin and colors. No standard of quality control has been set.

Macamides and maceaene were considered as the unique substance in the plant of maca. Seven, six , and five macamides were identified from the petrol ester extract of black, yellow, and purple maca in the present study, respectively (Table 1). Macamide variations and contents in the maca with the same skin colors was the uniform. The variations and contents of macamides are only dependent on skin colors of maca. By far, the maca was only cultivated in Yunnan province due to the high altitude and unique climatic conditions, in China. Although the samples were collected at random and the specific origin of the plant materials was not confirmed, the maca was ascertained to be from Yunnan Province. It was possible that the variations and contents of macamides were not affected in the maca with the same skin colors since the natural environment for cropping maca was similar. Maceaene was found in all the tested nine samples and the contents differentiation was negligible (Table 1). Whether the minute differentiation of maceaene was caused by sample or cropping environment was not confirmed since no control was supplied in the present study. Macamides belonged to the derivatives of fatty acid, which was known as antioxidant substance. In the present study, we hypothesized that macamides in the petrol ester extract was contributed to antioxidant activity $[28,29]$.

STZ diabetic rats was generally developed into typical diabetic complications [30]. Various proteins, including haemoglobin, albumin, collagen, LDL or crystalline proteins undergo nonenzymatic glycation [31], which may induce oxdative stresses by the formation of oxygenderived free radicals under diabetic condition [32]. In the present study, the administration of PM YM, and BM extracts all decreased plasma glucose levels in DM rats and prevented STZ-induced oxidative stress (Figure1). This suggested that maca could ameliorate the oxidative stress caused by hyperglycaemia.

The concentrations of lipid peroxides in the tissues are low under physiological conditions. Elevated levels of lipid peroxides in the plasma of diabetic rats was observed [33], which is a free-radicalinduced process due to oxidative deterioration of PUFA and resulted in the development of both type I and II diabetes [34]. The most commonly used indicators of lipid peroxidation are TBARS products [35]. The increased lipid peroxidation in the tissues of diabetic animals may be due to the observed increase in the concentration of TBARS in the liver and kidney of diabetic rats [36]. Our results showed that the levels of TBARS in D-CK animals were higher in liver tissue than those of $\mathrm{N}$ $\mathrm{CK}$ group, and were restored to normal values after the treatment with maca extract of PM , BM, and YM (Figure 2).

$\mathrm{CP}$ is another feature of irreversible oxidative damage, often leading to a loss of protein function, which is considered as a widespread indicator of severe oxidative damage and disease-derived protein dysfunction. STZ-induced oxidative damage in proteins was revealed by the increased content of CP in the plasma and liver tissue [37]. The treatment with three kinds of maca extracts all lowered the contents of $\mathrm{CP}$ in liver tissues of DM rats (Figure 3). The decreased levels in PM group were more notable than those in BM and YM group. Oxidative stress in diabetes coexists with a reduction in the antioxidant capacity, which can increase the deleterious effects of the free radicals. SOD protects tissues against oxygen free radicals by catalysing the removal of superoxide radical, converting it into $\mathrm{H}_{2} \mathrm{O}_{2}$ and molecular oxygen, which both damage the cell membrane and other biological structures [38]. Catalase is a haemprotein and responsible for the detoxification of significant amounts of $\mathrm{H}_{2} \mathrm{O}_{2}$ [39]. Reduced activities of SOD and catalase in the liver and pancreas during diabetes were reported, resulting in a number of deleterious effects due to the accumulation of superoxide radicals and hydrogen peroxide [40]. The activities of SOD and CAT of RBC as well as SOD of liver were significantly increased after oral administration of BM extract. YM extract can only enhance SOD activity in liver. On the other hand, PM extract significantly enhanced SOD and CAT activity of both RBC and liver (Table 2). The DM rats treated with maca extracts showed the reduced TBARS and CP levels, which may be associated with an increased activities of SOD and CAT. GSH is a major intracellular non-protein sulphydral compound and is accepted as the most important intracellular hydrophilic antioxidant [41]. Also, GSH acts as a co-substrate for GPx activity and as a co-factor for 
many enzymes and stress resistance of many cells is associated with the elevation of GSH levels. A decreased GSH content may predispose the cells to a lower defense against condition of oxidative stress during several degenerative disease conditions including diabetes [42]. The GSH contents were increased only after oral administration of PM extract in the present study (Figure 4). This indicated that DM rats model achieved higher antioxidant defense against STZ-induced oxidative stress.

\section{CONCLUSION}

Although the petroleum ether extract of maca with different skin colors showed antioxidant ability to DM rats, no correlation of the contents of macamides with antioxidant ability was observed. Some other compounds may be also attributed to the antioxidant activity. However, as a whole, the PM extract possessed the most potent antioxidant ability among the three species of maca.

\section{ACKNOWLEDGEMENTS}

The study was supported by doctoral grant (20124404110018) in China

\section{REFERENCES}

1. Giugliano D, Ceriello A, Paolisso G. Oxidative stress and diabetic vascular complications. Diabetes Care,1996. 19: p. 257-267.

2. Baynes JW. Role of oxidative stress in development of complications in diabetes. Diabetes, 1991. 40: p. 405-412.

3. McLennan SV, Heffernan S, Wright L, Rae C, Fisher $\mathrm{E}$, Rue DK, Turtle JR. Changes in hepatic glutathione metabolism in diabetes. Diabetes, 1991. 40: p. 344-348.

4. Mullarkey CJ, Edelstein D, Brownlee M. Free radical generation by early glycation products: a mechanism for accelerated atherogenesis in diabetes. Biochem Biophys Res Commun, 1990. 173: p. 932-938.

5. Strain JJ. Disturbances of micronutrient and antioxidant status in diabetes. Proc Nutr Soc, 1991. 50: p. 591-604.

6. Soto C, Recoba R, Barron H, Alvarez C, Favari L. Silymarin increases antioxidant enzymes in alloxan-induced diabetes in rat pancreas. Comp
Biochem Physiol C Toxicol Pharmacol, 2003. 136: p. 205-212.

7. Szkudelski T. The mechanism of alloxan and streptozotocin action in beta-cells of the rat pancreas. Physiol Res, 2001. 50: 536-546.

8. Valentova K, Frcek J, Ulrichova J. Yacon, (Smallanthu sonchifolius) and Maca (Lepidium meyenii), traditional Andean crops as new functional foods on the European market. Chemi listv, 2001. 95: p. 594-601.

9. Leon J. The 'Maca' (Lepidium meyenii), a little known food plant of Peru. Econ Bot. 1964. 18: p. 122-127.

10. Kilham C: Tables from the medicine trail: tracking down the health secrets of shamans, herbalists, mystics, yogis and other healers. Rodale Press, Emmaus, PA; 2000. p. 182-215.

11. Zheng BL, He K, Rogers L, Shao Y, Huang ZY, Yan SJ, Qien LC, Zheng QY. Effect of a lipidic extract from Lipidium menyenii on sexual behavior in mice and rats. Urology, 2000. 55: p. 598-602.

12. Cicero AFG, Bandieri E and Arletti R. Lepidium meyenii walp. improves sexual behavior in male rats independently from its action on spontaneous locomotor activity. J Ethnopharmacol, 2001.75: p. 225-229.

13. Gonzales GF, Rubio J, Chung A, Gasco M and Villegas L. Effect of alcoholic extract of lipidium meyenii (Maca) on testicular function in male rats. Asian J Androl, 2003. 5: p. 349-352.

14. Gonzales GF, Gasco M, Cordova A, Chung A, Rubio $\mathrm{J}$ and Villegas L. Effect of Lipidium meyenii (Maca) on spermatogenesis in male rats acutely exposed to high altitude (4340 m). J Endocrinol, 2004. 180: p. 87-95.

15. Chung F, Rubio J, Gonzales C, Gasco M and Gonzales GF. Dose-response effects of Lepidium meyenii (Maca) aqueous extract on testicular function and weight of different organs in adult rats. J Ethnopharmacol, 2005. 98: p. 143-147.

16. Quiros CF and Aliaga RC. Andean roots and tubers: ahipa, arracacha, Maca and yacon, herman $M$, Heller J (eds.). Rome: International Plant Genetic Resources Institute,1997. p. 174-197.

17. Valerio LG and Gonzales GF. Toxicological aspects of the South American herbs cat's claw (Uncaria tomentosa) and Maca (Lepidium meyenii): a critical synopsis. Toxicol Rev, 2005. 24: p. 11-35,

18. Chen XQ, Wang QQ, Li JM, Wang YQ, Zhang XZ, $\mathrm{Li}$ K. Comparison of simultaneous distillation and extraction, static headspace and headspace-solid phase microextraction coupled with GC/MS to measure the flavour components of Tricholoma matsutake. Asian J Chem, 2013. 25: p. 6059-6063.

19. McCollom MM, Villinski JR, McPhail KL, Craker LE, Gafner S. Analysis of Macamides in samples of Maca (Lepidium meyenii) by HPLC-UV-MS/MS. Phytochem Anal, 2005.16: p. 463-469. 
20. Ganzera M, Zhao J, Muhammad I, Khan IA. Chemical profiling and standardization of Lepidium meyenii (Maca) by reversed phase high performance liquid chromatography. Chem Pharm Bull, 2002. 50: p. 988-991.

21. Clement C, Diaz D, Manrique I, Avula B, Khan IA, Ponce A, Dante D, Kunz C, Mayer AC, Kreuzer M. Secondary metabolites in Maca as affected by hypocotyl color, cultivation history, and site. Agronomy J, 2010. 102: p. 431-439.

22. Gonzales GF, Miranda S, Nieto J, Fernandez G, Yucra S, Rubio J, Yi P, Gasco M. Red Maca (Lepidium meyenii) reduced prostate size in rats. Reprod Biol Endocrinology, 2005. 3: doi:10.1186/1477-7827-3-5.

23. Ohkawa H, Ohishi N, Yagi K. Assay for lipid peroxides in animal tissues by thiobarbituric acid reaction. Anal Biochem, 1979. 95: p. 351-358.

24. Chain FE, Grau A, Martins JC, Catalan CAN. Macamides from wild 'Maca', Lepidium meyenii Walpers (Brassicaceae). Phytochem Lett, 2014. 8: p. 145-148.

25. Wu H, Kelley CJ, Pino-Figueroa A, Vu HD, Maher TJ. Macamides and their synthetic analogs: evaluation of in vitro FAAH inhibition. Bioorg Med Chem, 2013. 21: p. 5188-5197.

26. Wang Y, Wang Y, McNeil B, Harvey LM. Maca: an Andean crop with multi-pharmacological functions. Food Res Int, 2007. 40: p. 783-792.

27. Muhammad I, Zhao J, Dunbar DC, Khana IA. Constituents of Lepidium meyenii 'Maca' . Phytochemistry, 2002. 59: p. 105-110.

28. Fan L, Ma J, Chen YH, Chen XQ. Antioxidant and antimicrobial phenolic compounds from Setaria viridis. Chem Nat Comp, 2014. 50: p. 433-437.

29. Wang LW, Liang J, Wang XD, Yuan XF, Zhao B, Yang YW. High efficient antioxidant activity of extracts from Lepidium meyenii Walp. Asian $\mathbf{J}$ Chem, 2012. 24: p. 4795-4798.

30. Ozturk Y, Altan VM and Yildizoglu A. Effect of experimental diabetes and insulin on smooth muscle functions. Pharmacol Rev, 1996. 48: p. 69112.

31. Klein, R. Hyperglycemia and microvascular and macrovascular disease in diabetes. Diabetes Care, 1995. 18: p. 258-268.

32. Gupta BL, Nehal M, Baquer NZ. Effect of experimental diabetes on the activities of hexokinase, glucose-6-phosphate dehydrogenase and catecholamines in rat erythrocytes of different ages. Indian J Exp Biol, 1997. 35: p. 792-795.

33. Karpen CW, Pritchard KA, Merola AJ, Panganamala RV. Alterations of the prostacyclin-thromboxane ratio in streptozotocin induced diabetic rats. Prostaglandins Leukot Med, 1982. 8: p.93-103.

34. Metz SA. Oxygenation products of arachidonic acid: third messengers for insulin release. Prostaglandins, 1984. 27: p.147-151.
35. Lyons TJ. Oxidized low-density lipoproteins, a role in the pathogenesis of atherosclerosis in diabetes. Diabet Med, 1991. 8: p. 411-419.

36. Stanely P, Prince M, Menon VP. Antioxidant action of Tinospora cordifolia root extract in alloxan diabetic rats. Phytother Res, 2001. 15: p. 213-218.

37. Cumaoglu A, Cevik C, Rackova L, Ari N, Karasu C. Effects of antioxidant stobadine on protein carbonylation, advanced oxidation protein products and reductive capacity of liver in streptozotocin-diabetic rats: role of oxidative/nitrosative stress. Biofactors, 2007. 30: p. 171-178.

38. Arivazhagan P, Thilagavathy T, Pannerselvam C. Antioxidant lipoate and tissue antioxidants in aged rats. J Nutr Biochem, 2000. 11: p. 122-127.

39. Cheng L, Kellogg EW, Packer L. Photoactivation of catalase. Photochem Photobiol, 1981. 34: p. 169174 ,

40. Searle AJ, Wilson RL. Glutathione peroxidase: effect of superoxide, hydroxyl and bromine free radicals on enzyme activity. Int J Rad Biol, 1981. 34: p. 125-129.

41. Melov SA. Animal models of oxidative stress, aging and therapeutic antioxidant interventions. Int $\mathrm{J}$ Biochem Cell Biol, 2002. 34: p. 1395-1400.

42. Hussein MA. Antidiabetic and antioxidant activity of Jasonia montana extract in streptozotocininduced diabetic rats. SPJ, 2008. 16: p. 214-221.

Received: January 15, 2016; Accepted: April 25, 2016 